\title{
Electric polarizability of the neutron in dynamical quark ensembles
}

\section{Michael Engelhardt*}

Department of Physics, New Mexico State University

E-mail: engel @nmsu. edu

The background field method for measuring the electric polarizability of the neutron is adapted to the dynamical quark case, resulting in the calculation of (certain space-time integrals over) threeand four-point functions. Particular care is taken to disentangle polarizability effects from the effects of subjecting the neutron to a constant background gauge field; such a field is not a pure gauge on a finite lattice and engenders a mass shift of its own. At a pion mass of $m_{\pi}=759 \mathrm{MeV}$, a small, slightly negative electric polarizability is found for the neutron.

The XXV International Symposium on Lattice Field Theory

July 30 - August 4, 2007

Regensburg, Germany

* Speaker. 


\section{Introduction}

The response of hadrons to external electromagnetic fields is characterized by their polarizabilities. Polarizabilities manifest themselves, e.g., in soft Compton scattering; the incoming electromagnetic wave distorts the hadron, and this distortion in turn leads to a modified scattered wave. Accordingly, polarizabilities appear as effects of second order in the external field in the effective hadron Hamiltonian. In a low-energy expansion of that Hamiltonian, the leading term embodies the hadron mass shift in constant electric and magnetic fields $E$ and $B$,

$$
H_{\mathrm{eff}}=\frac{1}{2}\left(\alpha E^{2}+\beta B^{2}\right)
$$

with the static electric and magnetic polarizabilities $\alpha$ and $\beta$, respectively. Carrying the low-energy expansion to subsequent orders, dependences on particle spin and electromagnetic field gradients appear [1].

The work presented here is concerned specifically with the static electric polarizability $\alpha$ of the neutron. There are two chief new elements to this investigation. On the one hand, dynamical quark ensembles are used; on the other hand, the role of constant background gauge fields is recognized and their effects are properly taken into account. As far as the former issue is concerned, it should be noted that, when introducing an explicit external electromagnetic field to compute polarizabilities on the lattice, the transition from quenched to dynamical quark ensembles entails more than just the usual increased computational cost of generating the ensemble. In the quenched approximation, the backreaction of the quark fields on the gluonic degrees of freedom is truncated; thus, the external electromagnetic field, which in turn only couples to the quark fields, does not influence the gauge ensemble. As a result, one can generate the ensemble in the absence of the background field and introduce the latter a posteriori by a suitable modification of the link variables. By contrast, in the dynamical quark case, at first sight, one would need to recompute a new gauge ensemble for every specific external field considered, a prohibitively expensive approach if directly applied. Previous quenched investigations $[2,3,4]$ were thus able to take advantage of considerable simplifications not available for dynamical quarks. The dynamical quark case necessitates significant modifications of the computational scheme in order to become tractable, cf. section 2 below.

Concerning the issue of constant background gauge fields, consider introducing a constant electric field in the 3-direction via the gauge field

$$
A_{3}(t)=E\left(t-t_{0}\right) \equiv A+E t .
$$

For given electric field $E$, one still has a continuum of choices for $t_{0}$ or, equivalently, $A$. In a setting in which the spatial directions are infinite, this is merely a gauge freedom; $A$ can be arbitrarily shifted by gauge transformations. However, if the spatial directions are finite, such as in a lattice calculation, this gauge freedom is reduced by the need to preserve the boundary conditions; as a consequence, physics in general depends on the value of $A$. As a simple example, consider a charged particle on a circle of length $L$ with periodic boundary conditions in a constant gauge field $A$. The Hamiltonian is $H=(p-A)^{2}$ and the energy levels are $E_{n}=(2 \pi n / L-A)^{2}$, where $n$ is any whole number. In particular, the ground state energy as a function of $A$ is plotted in Fig. 1. Only a discrete symmetry under shifts $A \rightarrow A+2 \pi / L$ remains; $A$ can be interpreted as a Bloch momentum of the charged particle. 


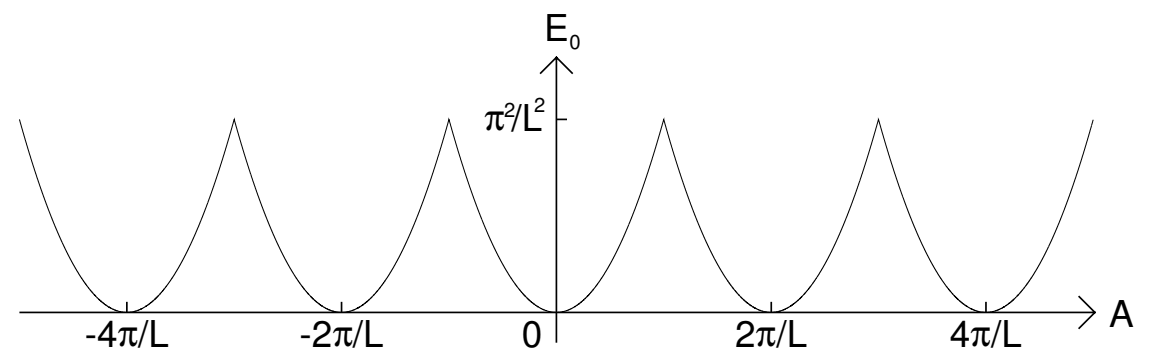

Figure 1: Ground state energy of a charged particle on a circle of length $L$ with periodic boundary conditions, as a function of the constant gauge field $A$ to which it is subjected, cf. main text.

Similarly, the mass shift of a neutron in the external field (1.2) is a function of both the parameters $E$ and $A$. The dependence on $A$ is a dominant effect which must disentangled from the polarizability in presence of the electric field $E$.

\section{Perturbative expansion}

As mentioned above, in the presence of dynamical quarks, the gauge ensemble depends on the specific external electromagnetic field under consideration. However, it would be prohibitively expensive to recompute the ensemble for each background field. Relief is provided by the fact that it is not necessary to be able to evaluate the neutron mass shift in arbitrary backgrounds; after all, the electric polarizability is given specifically by the second Taylor coefficient of that mass shift as a function of the electric field, cf. (1.1). To evaluate a Taylor coefficient, it is sufficient to consider only infinitesimal external fields, and in this case, one can correspondingly expand the neutron two-point function determining the mass shift. Introducing the external field $A_{3}$, cf. (1.2), into the link variables as an additional $U(1)$ phase,

$$
U_{3} \longrightarrow \exp \left(i \int d x_{3} \cdot A_{3}\right) \cdot U_{3}=\left(1+i a A_{3}-a^{2} A_{3}^{2} / 2+\ldots\right) \cdot U_{3},
$$

one obtains an action

$$
S=S_{0}+S_{\text {ext }}
$$

composed of the QCD action in the absence of the external field, $S_{0}$, and the coupling to the external field

$$
\begin{aligned}
S_{\text {ext }}=z_{V} \frac{1}{2} \Sigma_{x} \bar{\psi}(x)\left[\left(i a A_{3}\right.\right. & \left.\left.-a^{2} A_{3}^{2} / 2\right) \cdot U_{3}(x) \cdot\left(-1+\gamma_{3}\right) \cdot \psi\left(x+e_{3}\right)\right) \\
& \left.\left.+\left(i a A_{3}+a^{2} A_{3}^{2} / 2\right) \cdot U_{3}^{\dagger}\left(x-e_{3}\right) \cdot\left(1+\gamma_{3}\right) \cdot \psi\left(x-e_{3}\right)\right)\right],
\end{aligned}
$$

which has been written here specifically for Wilson-type four-dimensional quark fields. The particular computational scheme adopted in the following uses domain wall fermions in the valence sector; however, quark propagators are evaluated starting from sources which only have support on the four-dimensional domain walls, and they are projected back onto four dimensions at the sink. This is a compromise due to hardware constraints on the storage of propagators; correspondingly, the coupling to the external field is effected via the four-dimensional projected fields $\psi$, as written 
in (2.3). Concomitantly, it is necessary to allow for the renormalization factor $z_{V}$ on quark bilinears to compensate for the effect of the projection. This factor was determined in [5] by evaluating the number of valence quarks in the neutron, yielding $z_{V}=1.12 \pm 0.12$. Inserting the action (2.2) into the neutron two-point function, one has

$$
\begin{array}{r}
\left\langle N_{\beta}(y) \bar{N}_{\alpha}(x)\right\rangle=\int[D U][D \psi][D \bar{\psi}] \exp \left(-S_{0}\right)\left(1-S_{\text {ext }}+S_{\text {ext }}^{2} / 2+\ldots\right) N_{\beta}(y) \bar{N}_{\alpha}(x) / \\
\int[D U][D \psi][D \bar{\psi}] \exp \left(-S_{0}\right)\left(1-S_{\text {ext }}+S_{\text {ext }}^{2} / 2+\ldots\right)
\end{array}
$$

To obtain specifically the part of the two-point function which is quadratic in the external field, one therefore must calculate neutron two-point functions with either two additional insertions of the vertex linear in the external field, cf. (2.3), or one additional insertion of the vertex quadratic in the external field, cf. (2.3), as displayed in Fig. 2.

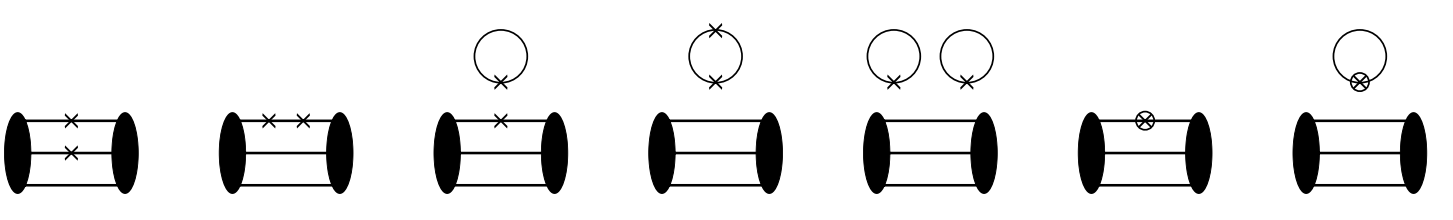

Figure 2: Diagrams of second order in the external field resulting from inserting the electromagnetic vertices generated by the interaction term $S_{\text {ext }}$ into a neutron two-point function. Crosses denote vertices linear in the background field, circled crosses vertices quadratic in the external field.

The space-time positions of the insertions are to be integrated over according to (2.3); thus, one is effectively calculating certain space-time integrals over three- and four-point functions. These functions are evaluated in the unperturbed ensemble generated by the action $S_{0}$. The two right-most diagrams involving the vertex quadratic in the external field act as contact terms renormalizing the second and fourth diagrams from the left, respectively. As usual, the denominator of (2.4) effects the subtraction of statistically disconnected pieces from diagrams containing disconnected quark loops.

\section{Extracting the mass shift}

Focusing on the zero-momentum, unpolarized component of the neutron two-point function,

$$
G(p=0, t)=\Sigma_{\vec{y}} \operatorname{Tr}\left(\frac{1+\gamma_{0}}{2}\langle N(y) \bar{N}(x)\rangle\right) \stackrel{t \rightarrow \infty}{\longrightarrow} W \exp (-m t)
$$

one observes an exponential decay in time at a rate governed by the neutron mass $m$; $W$ characterizes the overlap between neutron sources/sinks and the neutron ground state wave function. In the setting discussed here, some care must be exercised in interpreting the two-point function [5]. The Hamiltonian in the presence of the external field (1.2) is time-dependent, since a translation in time is equivalent to a shift in the constant gauge field $A$, cf. (1.2); on the other hand, a change in $A$ modifies the physical spectrum. For infinitesimal external fields, this time dependence can be treated adiabatically, i.e., the exponential decay of the two-point function can still be interpreted in terms of the neutron mass, which, however, will continuously adjust as the external field changes. 
Expanding the overlap $W$ and the neutron mass $m$ in terms of the external field parameters $A$ and $E$,

$$
\begin{aligned}
W & =W_{0}+W^{(1)}(A, E)+W^{(2)}(A, E)+\ldots \\
m & =m_{0}+m^{(2)}(A, E)+\ldots
\end{aligned}
$$

where the superscripts denote the order in the external parameters, the second-order part of $G(p=0, t)$ behaves as

$$
G^{(2)}(p=0, t) \stackrel{t \rightarrow \infty}{\longrightarrow} W_{0} \exp \left(-m_{0} t\right)\left(\frac{W^{(2)}(A, E)}{W_{0}}-m^{(2)}(A, E) t\right) .
$$

Note that, in (3.3), use has been made of the fact that there can be no first-order term in the neutron mass in the absence of a nontrivial $\theta$-angle. Thus, dividing (3.4) by the unperturbed correlator $W_{0} \exp \left(-m_{0} t\right)$ yields a ratio whose slope as a function of time, $S(A, E)$, corresponds, up to a minus sign, to the mass shift of the neutron in the presence of the external electromagnetic field,

$$
-S(A, E)=m^{(2)}(A, E) \equiv m_{2}^{A A} A^{2}+m_{2}^{A E} A E+m_{2}^{E E} E^{2} .
$$

Here, again, care must be taken in interpreting the results. As mentioned above, the spectrum of the system adjusts adiabatically to the external field, i.e., $m^{(2)}$ and $W^{(2)}$ themselves in general are time-dependent. This complicates the interpretation of $S$ in terms of the mass shift $m^{(2)}$. However, at the particular point where the physical spectrum is stationary in $A$, the time dependence of $m^{(2)}$ and $W^{(2)}$ is of at least quadratic order. This is due to the equivalence between shifts in $A$ and shifts in time, cf. (1.2); stationarity in $A$ implies stationarity in time. Thus, at the stationary point only, which in practice is identified by seeking out the extremum of the slope $S(A, E)$ as a function of $A$, one can indeed directly interpret that slope as giving $m^{(2)}=-S$.

In turn, as discussed in more detail in [5], the part of the neutron mass shift due specifically to its electric polarizability can be isolated from the combined dependence of $m^{(2)}$ on $E$ and $A$ by seeking out the extremum of $m^{(2)}(A, E)$ (or, equivalently, $S(A, E)$ ) as a function of $A$. The mass shift obtained at the extremum then yields the electric polarizability via

$$
\alpha=-\left.\frac{2}{E^{2}} m^{(2)}\right|_{\text {extremum }}
$$

cf. (1.1). To carry out this program in practice, one thus needs measurements at three different values of $A$ (or, equivalently, $t_{0}$ ) in (1.2), while $E$ is fixed. This then defines a parabola in $t_{0}$, permitting the extraction of the extremum as a function of $t_{0}$.

\section{Measurements}

The program described above was put into practice using 99 configurations from one of the dynamical 2+1 flavor Asqtad quark ensembles provided by the MILC collaboration [6]. Specifically, the $S U$ (3) flavor-symmetric ensemble with quark masses $a m_{u}=a m_{d}=a m_{s}=0.05$ was utilized, where $a=0.124 \mathrm{fm}$ denotes the lattice spacing. This choice of quark masses corresponds to a pion mass of $m_{\pi}=759 \mathrm{MeV}$. On the other hand, as already mentioned in section 2, in the valence sector, 


\begin{tabular}{|c||c|c|}
\hline & $\begin{array}{c}\text { connected } \\
\text { diagrams }\end{array}$ & $\begin{array}{c}\text { all } \\
\text { diagrams }\end{array}$ \\
\hline \hline $\begin{array}{c}S /\left(a^{3} E^{2}\right) \\
t_{0}=-10 a\end{array}$ & $0.46(18)$ & $0.26(26)$ \\
\hline $\begin{array}{c}S /\left(a^{3} E^{2}\right) \\
t_{0}=0\end{array}$ & $0.000(16)$ & $-0.033(43)$ \\
\hline $\begin{array}{c}S /\left(a^{3} E^{2}\right) \\
t_{0}=6 a\end{array}$ & $-0.017(3)$ & $-0.037(25)$ \\
\hline \hline $\begin{array}{c}-m^{(2)} /\left(a^{3} E^{2}\right) \\
(\mathrm{extremum})\end{array}$ & $-0.034(6)$ & $-0.052(24)$ \\
\hline
\end{tabular}

Table 1: Measurements of the slope $S(A, E)$, together with a determination of its extremum as a function of $A$. The electric field $E$ is cast in Gaussian units. Errors were obtained by the jackknife method, including the fluctuations of the renormalization constant $z_{V}$.

domain wall quarks were used; this hybrid scheme has been previously employed in $[7,8]$, where also details concerning parameter tuning in this approach can be found.

Table 1 displays the measurements obtained for the slope $S(A, E)$, together with a determination of its extremum as a function of $A$, which yields (up to a minus sign) the mass shift due to the electric polarizability of the neutron. In all measurements, the neutron source was placed at $t=0$ and the slope $S(A, E)$ was extracted by fitting in the range $4 a \leq t \leq 10 a$. The disconnected traces were evaluated using stochastic estimation with 120 complex $Z(2)$ stochastic sources (240 for the case $t_{0}=-10 a$, which displayed particularly strong statistical fluctuations). Using (3.6) to obtain the electric polarizability of the neutron from the last line of Table 1 , one finally arrives at ${ }^{1}$

$$
\alpha=(-2.0 \pm 0.9) \cdot 10^{-4} \mathrm{fm}^{3}
$$

at a pion mass of $m_{\pi}=759 \mathrm{MeV}$.

\section{Discussion}

Compared to the experimental value reported by the Particle Data Group [9], namely, $\alpha=$ $(11.6 \pm 1.5) \cdot 10^{-4} \mathrm{fm}^{3}$, the result (4.1) implies a strong variation with pion mass. Such a variation is indeed expected from Chiral Effective Theory [10, 11, 12]. Fig. 3 displays the result (4.1) in relation to the pion mass dependence expected from the "Small Scale Expansion" approach [10, 11], a systematic extension of leading-one-loop Heavy Baryon Chiral Perturbation Theory by explicit $\Delta$ degrees of freedom. While at a pion mass of $m_{\pi}=759 \mathrm{MeV}$, Chiral Effective Theory cannot be taken as more than a qualitative hint, the comparison displayed in Fig. 3 is tantalizing and calls for a push towards lower pion masses, where a quantitative connection with Chiral Effective Theory can be made. In view of the computational effort which was required for the present dynamical quark calculation at $m_{\pi}=759 \mathrm{MeV}$, such further progress is feasible, but will require significant resources by current standards.

\footnotetext{
${ }^{1}$ Cf., however, the "Note added in proof" of ref. [5].
} 


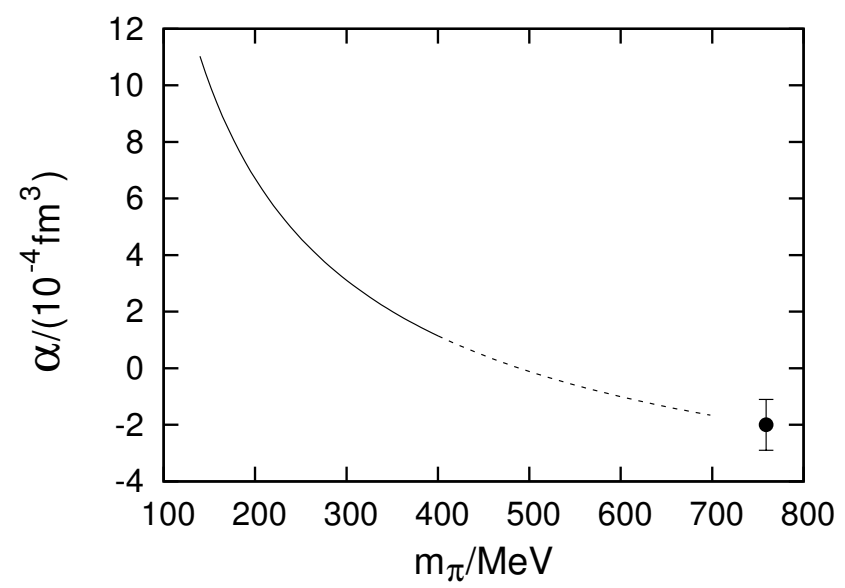

Figure 3: Comparison of the lattice measurement of the electric polarizability $\alpha$ of the neutron obtained in this work with the pion mass dependence expected from the "Small Scale Expansion" approach [10, 11]. This Chiral Effective Theory is expected to be accurate up to pion masses of about $300-400 \mathrm{MeV}$ (solid line); the dashed line is obtained if one naively continues the Chiral Effective Theory expression to higher pion masses, beyond its realm of controlled quantitative applicability.

\section{Acknowledgments}

This investigation benefited from various exchanges with R. Brower, M. Burkardt, W. Detmold, R. Edwards, H. Grießhammer, D. B. Kaplan, J. Negele, K. Orginos, J. Osborn, D. Renner, M. Savage, D. Toussaint and W. Wilcox. Computations were carried out using resources provided by the U.S. DOE through the USQCD project at Jefferson Lab. This work is supported by the U.S. DOE under grant number DE-FG03-95ER40965.

\section{References}

[1] D. Babusci, G. Giordano, A. I. L’vov, G. Matone and A. M. Nathan, Phys. Rev. C 58 (1998) 1013.

[2] H. R. Fiebig, W. Wilcox and R. M. Woloshyn, Nucl. Phys. B324 (1989) 47.

[3] J. Christensen, F. X. Lee, W. Wilcox and L. Zhou, Nucl. Phys. Proc. Suppl. 119 (2003) 269.

[4] J. Christensen, W. Wilcox, F. X. Lee and L. Zhou, Phys. Rev. D 72 (2005) 034503.

[5] M. Engelhardt (LHPC Collaboration), Phys. Rev. D 76 (2007) 114502.

[6] C. W. Bernard, T. Burch, K. Orginos, D. Toussaint, T. A. DeGrand, C. DeTar, S. Datta, S. A. Gottlieb, U. M. Heller and R. Sugar, Phys. Rev. D 64 (2001) 054506.

[7] R. G. Edwards, G. T. Fleming, P. Hägler, J. W. Negele, K. Orginos, A. Pochinsky, D. B. Renner, D. G. Richards and W. Schroers (LHPC Collaboration), Phys. Rev. Lett. 96 (2006) 052001.

[8] P. Hägler, W. Schroers, J. Bratt, R. G. Edwards, M. Engelhardt, G. T. Fleming, B. Musch, J. W. Negele, K. Orginos, A. V. Pochinsky, D. B. Renner and D. G. Richards (LHPC Collaboration), arXiv:0705.4295.

[9] W.-M. Yao et al., J. Phys. G: Nucl. Part. Phys. 33 (2006) 1.

[10] T. R. Hemmert, B. R. Holstein and J. Kambor, Phys. Rev. D 55 (1997) 5598.

[11] R. P. Hildebrandt, H. W. Grießhammer, T. R. Hemmert and B. Pasquini, Eur. Phys. J. A 20 (2004) 293.

[12] W. Detmold, B. C. Tiburzi and A. Walker-Loud, Phys. Rev. D 73 (2006) 114505. 